\title{
INTERVENCIONES BASADAS EN CAMBIO DE HÁBITOS PARA UNA PÉRDIDA DE PESO SOSTENIBLE
}

\section{HABIT-BASED INTERVENTIONS FOR SUSTAINABLE WEIGHTLOSS}

\section{Pág. 12,13}

Recibido: 01-02-2020

Vanessa Medina Peñaloza ${ }^{1}$

Aceptado: 15-03-2020

1.Licenciada en nutrición humana y dietética, CPN 2219-16

Docente en la Universidad de Ciencias Médicas UCIMED, San José, Costa Rica

\section{Resumen}

Las dietas tradicionales han mostrado ser poco efectivas a largo plazo, por su naturaleza restrictiva y por no proveer al paciente de herramientas que le ayuden a trabajar sobre otras áreas de su vida, que influyen en su manera de alimentarse. Por el contrario, estudios recientes han demostrado el potencial de las intervenciones basadas en hábitos para influir positivamente en los comportamientos de salud y reducir el exceso de peso si fuera necesario.

Palabras clave: comportamiento, dieta, hábitos, pérdida de peso, sobrepeso.

\section{Abstract:}

Traditional diets have proven to be ineffective in the long term due to their restrictive nature and for not providing the patient with tools to help them work on other areas of their life that influence their way of eating. On the contrary, recent studies have demonstrated the potential of habitbased interventions to positively influence health behaviors and reduce excess weight if necessary.

Keywords: behavior, diets, habits, weightloss, overweight

\section{Desarrollo:}

De acuerdo con las cifras arrojadas por la Organización Mundial de la Salud, desde 1975 la obesidad se ha triplicado en más de 1900 millones

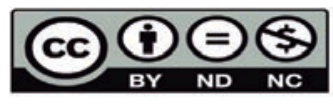

de adultos con sobrepeso, de los cuales poco más de un tercio presentan obesidad (OMS, 2018).

A pesar de toda la información que está disponible, el tratamiento sigue siendo de orden unifactorial, enfocado en qué y cuánto comer y, sin aportar una guía que permita alcanzar metas que sean permanentes y sostenibles a largo plazo. La idea de que las dietas restrictivas realizadas de manera independiente pueden ser exitosas por sí solas, ignora la realidad de que las personas comen por otras razones más allá de simplemente alimentarse.

Esto no es un concepto nuevo. Una publicación realizada en el año 2005 , hace referencia a una encuesta realizada en el año 1996, y desde entonces no hay evidencia convincente de que la dieta por sí sola produzca una pérdida de peso permanente. Esto se debe a que algunas comidas se hacen en respuesta a señales externas o como método para lidiar con estresores y estados emocionales negativos como depresión, ansiedad, enojo, aburrimiento o soledad (Abramson, 2005).

Las recomendaciones tradicionales para el control del peso generalmente se basan en dar indicaciones sobre qué cambios se deben hacer y por qué, lo cual requiere de acciones intencionales y conscientes para actuar. Sin embargo, la motivación para cambiar los comportamientos va disminuyendo, por lo que los cambios suelen ser de corta duración y se recupera el peso perdido. Estudios psicológicos muestran que los comportamientos habituales influyen fuertemente en los resultados de salud, por tanto, el potencial 
de las intervenciones basadas en el cambio de hábitos puede influir positivamente en los comportamientos de salud y reducir el exceso de peso (Cleo, Glasziou, Beller, Isenring, \& Thomas, 2018).

El control del peso a largo plazo es extremadamente difícil, debido a las interacciones entre la biología, comportamiento y el entorno obesogénico. Los cambios de comportamiento a largo plazo y el manejo del sobrepeso y la obesidad requieren atención continua. Las habilidades y estrategias conductuales específicas para el mantenimiento del peso ayudan a los pacientes a desarrollar una visión para el manejo a largo plazo, anticipar factores limitantes y preparar planes de contingencia, fatiga conductual moderada y, poner en perspectiva las inevitables fallas y recaídas de cualquier compromiso a largo plazo (Hall \& Kahan, 2018).

Una revisión sistemática y meta análisis publicada a finales del 2019 encontró diferencias estadísticamente significativas entre los grupos control y las intervenciones basadas en cambio de hábitos. Las intervenciones se enfocaban en crear nuevos hábitos (Ten Top Tips), romper hábitos viejos (Do Something Different) o una combinación de ambas (Transforming Your Life). Aquí se concluyó que los programas de pérdida de peso, basados en cambio o establecimiento de nuevos hábitos, son más efectivos para lograr una pérdida de peso clínicamente beneficiosa que solo los consejos enfocados en qué hacer (Cleo, Beller, Glasziou, Isenring, \& Thomas, 2019).

Los hábitos generalmente se forman repitiendo un comportamiento hasta que se vuelve más o menos automático. Una vez que se forma un hábito, se considera que la automaticidad es una herramienta poderosa, porque existe la seguridad de que el comportamiento se hará progresivamente más fácil. Por lo tanto, mantener la motivación y llevar a cabo constantemente el nuevo comportamiento solo es necesario hasta que se forme el hábito y el nuevo comportamiento se convierta en rutina. Romper hábitos no deseados se puede lograr mediante la reestructuración del entorno de un individuo o programando nuevas respuestas a ese entorno (Cleo, Beller, Glasziou, Isenring, \& Thomas, 2019).

\section{Conclusión}

Las intervenciones para la pérdida de peso basadas en el cambio de hábitos sugieren ser más efectivas a largo plazo, que las intervenciones tradicionales basadas únicamente en dieta y ejercicio, sobre todo si estas se realizan de manera aislada y sin ningún acompañamiento. Por lo tanto, sería importante realizar un cambio de paradigma a nivel profesional y empezar a utilizar estrategias más integrales que ayuden a los pacientes a salirse del círculo vicioso de las dietas que ofrecen resultados rápidos más no sostenibles.

\section{Bibliografía}

Abramson, E. (2005). On the Futility of Dieting. Medscape General Medicine, 31.

Cleo, G., Beller, E., Glasziou, P., Isenring, E., \& Thomas, R. (2019). Efficacy of habit-based weight loss interventions: a systematic review and meta -analysis. Journal of Behavior Medicine.

Cleo, G., Glasziou, P., Beller, E., Isenring, E., \& Thomas, R. (2018). Habit-based interventions for weight loss maintenance in adults with overweight and obesity: a randomized controlled trial. International Journal of Obesity, 374-383.

Hall, K., \& Kahan, S. (2018). Maintenance of lost weight and long-term management of obesity. The Medical Clinics of North America, 183-197.

OMS. (2018, Febrero 16). Obesidad y sobrepeso. Retrieved from Organización Mundial de la Salud: https://www.who.int/es/news-room/fact-sheets/ detail/obesity-and-overweight 